\title{
Optimal configuration of piezoelectric sensors and actuators for active vibration control of a plate using a genetic algorithm
}

Received: 6 December 2014 / Revised: 10 May 2015 / Published online: 11 June 2015

(C) The Author(s) 2015. This article is published with open access at Springerlink.com

\begin{abstract}
The purpose of this study is to suggest a new formulation for active vibration control of a rectangular plate based on the optimal positions/orientations of piezoelectric actuators/sensors attached to the plate. The free vibration and modal properties are derived by using Rayleigh-Ritz and the transient response by assumed modes methods based on the classical plate theory. Three criteria are proposed for optimal location of piezoelectric patches attached to the simply supported plate. In other words, the optimal positions/orientations of piezoelectric patches can be determined based on spatial controllability/observability gramians of the structure, as well as the consideration of residual modes to reduce the spillover effect. These criteria are used to achieve the optimal fitness function defined for a genetic algorithm optimizer to find the optimal locations/orientations of piezoelectric sensors/actuators. To control the vibrations of the plate, a negative velocity feedback control algorithm is designed. The results of simulations indicate that by locating piezoelectric patches in the optimal positions, the depreciation rate of the structure increases and the amplitudes of the plate vibrations reduce effectively. The effect of number of piezoelectric devices on the active damping property of the system is also analyzed.
\end{abstract}

\section{Introduction}

In recent years, the active vibration control has been an important challenge for spatial structures. The importance has been more remarkable and higher for larger and more flexible spatial structures. For vibration suppression, smart materials such as piezoelectric transducers may be used [1,2]. Therefore, determining smart actuator and sensor locations is a key subject for increasing the system efficiency. Kumar and Narayanan [3] determined the optimal locations of the sensor/actuator pair on a flexible beam. In this work, they used the linear quadratic regulator (LQR) strategy for active vibration control. Bruant et al. [4] proposed a method based on minimizing the mechanical energy integral of the system for obtaining the optimal actuator locations and maximizing the energy of the state outputs for obtaining the optimal sensor locations on the flexible beam. Hac and Liu [5] and Qiu et al. [6] developed an optimization method for finding the optimal locations of piezoelectric actuators and sensors based on the degree of observability and controllability. Sadri et al. [7] suggested

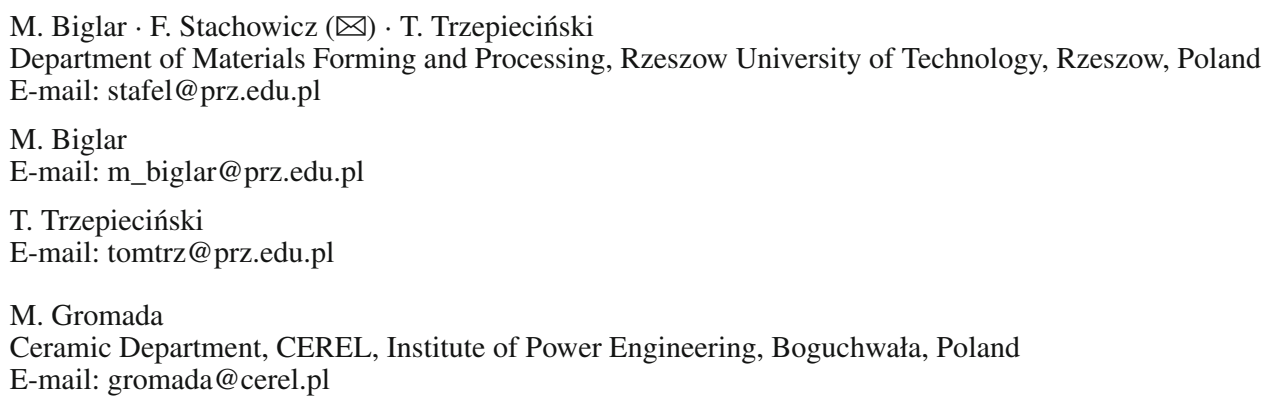


two criteria for obtaining the optimal locations of piezoelectric actuators using modal controllability and controllability gramian. Bruant et al. [8] and Han and Lee [9] determined the sensor and actuator locations with the consideration of controllability, observability, and spillover reduction. Furthermore, they used a genetic algorithm (GA) to find efficient locations of piezoelectric sensors/actuators. However, in these papers, the dynamic characteristics of both rectangular plate and piezoelectric sensors/actuators were not derived explicitly. Halim and Reza Moheimani [10] suggested a criterion for finding the optimal placement of collocated piezoelectric actuator/sensor pairs on a thin flexible plate. However, in this work, the motion equations were not derived analytically and the control algorithm was not used for suppressing the plate vibration. Yang and Lee [11] developed an integer-real-encoded GA to search for the optimal placement and size of the piezoelectric patches. Many control algorithms have been used to eliminate the vibrations, such as positive position feedback (PPF) control and direct velocity feedback control [12,13]. Yiqi and Yiming [14] established an analytical model for the active vibration control of a piezoelectric FGM plate based on a higher-order shear deformation plate and elastic piezoelectric theories. A finite element formulation was presented by $\mathrm{He}$ et al. [15], for the vibration control of a functionally graded plate (FGP) based on the classical lamination plate theory (CLPT). In the previous works of [14] and [15], the plates have been fully covered with integrated piezoelectric sensors and actuators. Thus, in these works, the optimization procedure was not required for an active vibration control.

In this study, we present a new formulation for combined active vibration control and optimal configuration of a rectangular plate by obtaining the optimal locations and orientations of piezoelectric actuators and sensors. Based on the CPT and linear piezoelectric theory, the equations of motion and sensor output equations of a smart plate are derived by using Hamilton's principle, the Rayleigh-Ritz approximation procedure, and the assumed modes method. For increasing the system efficiency, the optimal locations and orientations of piezoelectric actuators and sensors are determined based on spatial controllability and observability gramians and considering residual modes to reduce the spillover effect. The GA is utilized for optimizing the locations and orientations of piezoelectric devices. For active vibration control, a negative velocity feedback control algorithm is used. The remainder of the paper is organized as follows: We derive the basic equation in Sect. 2. In Sect. 3, we discuss the optimization of the location of sensors and actuators mounted on a rectangular plate. The genetic algorithm is used to find the optimal location of sensors and actuators in Sect. 4. In Sect. 5, designing a controller for active vibration of the plate and several simulations for showing the influence of optimization and the active control algorithm are outlined. Finally, in Sect. 6, we draw conclusions.

\section{Basic equations}

Consider a flexible plate (Fig. 1) with $\boldsymbol{N}_{\text {a }}$ piezoelectric actuators and $\boldsymbol{N}_{\text {se }}$ piezoelectric sensors. The total potential energy of the structure and piezoelectric patch is expressed as [16]:

$$
\begin{aligned}
\mathrm{PE}= & \int_{V^{\mathrm{st}}} \frac{1}{2} \mathbf{S}^{\mathbf{T}} \mathbf{c}_{\mathbf{s t}} \mathbf{S} \mathrm{d} V^{\mathrm{st}}+\sum_{j=1}^{N_{a}} \int_{V_{j}^{a}}\left(\frac{1}{2} \tilde{\mathbf{S}}^{\mathbf{T}} \mathbf{c}^{\mathbf{D}} \tilde{\mathbf{S}}-\tilde{\mathbf{S}}^{\mathbf{T}} \mathbf{h} \tilde{\mathbf{D}}^{\mathbf{j}}+\frac{1}{2} \tilde{\mathbf{D}}^{\mathbf{j} \mathbf{T}} \beta_{\mathbf{S}} \tilde{\mathbf{D}}^{\mathbf{j}}\right) \mathrm{d} V_{j}^{a} \\
& +\sum_{j=1}^{N_{\text {se }}} \int_{V_{j}^{\text {se }}}\left(\frac{1}{2} \tilde{\mathbf{S}}^{\mathbf{T}} \mathbf{c}^{\mathbf{D}} \tilde{\mathbf{S}}-\tilde{\mathbf{S}}^{\mathbf{T}} \mathbf{h} \tilde{\mathbf{D}}^{\mathbf{j}}+\frac{1}{2} \tilde{\mathbf{D}}^{\mathbf{j} \mathbf{T}} \beta_{\mathbf{S}} \tilde{\mathbf{D}}^{\mathbf{j}}\right) \mathrm{d} V_{j}^{\mathrm{se}}, \\
\tilde{\mathbf{S}}= & \mathbf{R}_{\mathbf{S}}^{\mathbf{j}} \mathbf{S}, \quad \tilde{\mathbf{D}}^{\mathbf{j}}=\mathbf{R}_{\mathbf{D}}^{\mathbf{j}} \mathbf{D}^{\mathbf{j}},
\end{aligned}
$$

where $\tilde{\mathbf{S}}$ and $\tilde{\mathbf{D}}^{\mathbf{j}}$ are strain and electric charge density vector in local coordinates, respectively. $\mathbf{C}_{\mathbf{s t}}, \mathbf{C}^{\mathbf{D}}$, $\mathbf{h}$, and $\beta_{\mathbf{S}}$ are the matrices of elastic constants of structure, elastic constants of piezoelectric patches under constant electric charge density, the piezoelectric constants, and the inverse of dielectric constant under constant strain; $\tilde{\mathbf{S}}^{\mathbf{T}}$ and $\tilde{\mathbf{D}}^{\mathbf{j} \mathbf{T}}$ are the transposes of $\tilde{\mathbf{S}}$ and $\tilde{\mathbf{D}}^{\mathbf{j}} . V^{\text {st }}, V^{\mathrm{a}}$, and $V^{\text {se }}$ are the volumes of structure, piezoelectric actuators, and piezoelectric sensors, respectively. $\boldsymbol{N}_{\mathrm{a}}$ and $\boldsymbol{N}_{\mathrm{se}}$ are the numbers of actuator and sensor patches.

By using the following relation, the local coordinate of system for each piezoelectric element can be transformed to global coordinates. $\boldsymbol{R}_{\boldsymbol{s}}^{j}$ and $\boldsymbol{R}_{\boldsymbol{d}}^{j}$ are strain and electric charge density transformation matrices, 


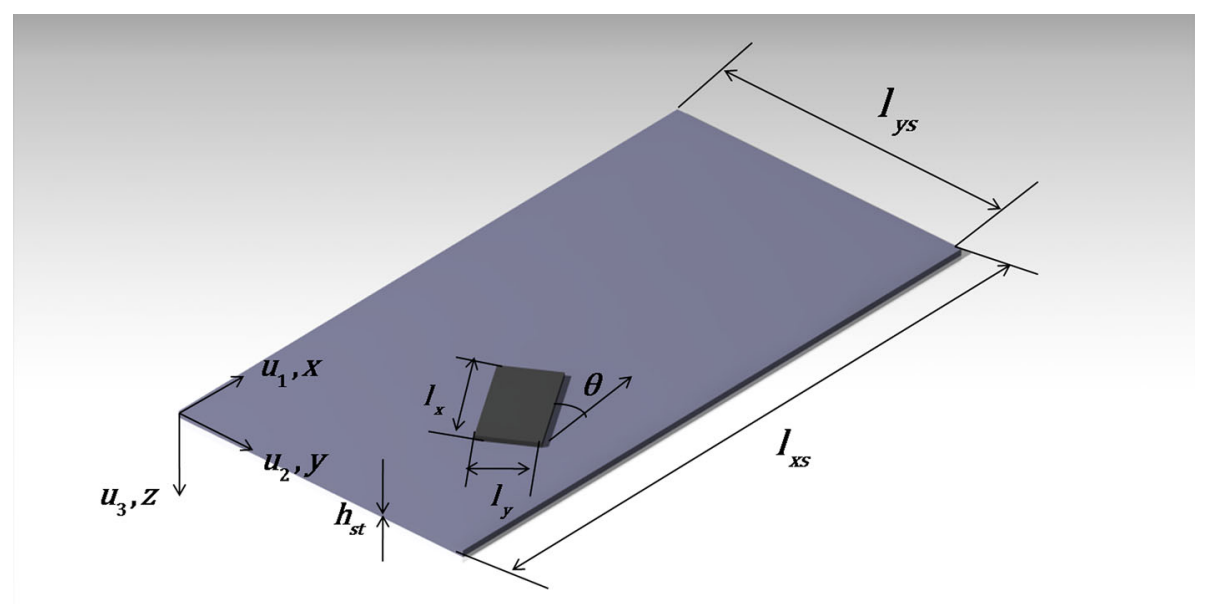

Fig. 1 Orientation of piezoelectric patches

respectively. The total kinetic energy of the structure and piezoelectric patches is obtained as [16]

$$
\begin{aligned}
\mathrm{KE}= & \frac{1}{2} \int_{V^{\mathrm{st}}} \rho^{\mathrm{st}}\left(\dot{u}_{1}^{2}+\dot{u}_{2}^{2}+\dot{u}_{3}^{2}\right) \mathrm{d} V_{\mathrm{st}} \\
& +\frac{1}{2} \sum_{j=1}^{N_{a}} \int_{V_{j}^{a}} \rho_{j}^{a}\left(\dot{u}_{1}^{2}+\dot{u}_{2}^{2}+\dot{u}_{3}^{2}\right) \mathrm{d} V_{j}^{a}+\frac{1}{2} \sum_{j=1}^{N_{\mathrm{se}}} \int_{V_{j}^{\mathrm{se}}} \rho_{j}^{\mathrm{se}}\left(\dot{u}_{1}^{2}+\dot{u}_{2}^{2}+\dot{u}_{3}^{2}\right) \mathrm{d} V_{j}^{\mathrm{se}} \\
& =\frac{1}{2} \int_{V^{\mathrm{st}}} \rho^{\mathrm{st}}\left(\dot{\mathbf{u}}^{\mathrm{T}} \dot{\mathbf{u}}\right) \mathrm{d} V_{\mathrm{st}}+\frac{1}{2} \sum_{j=1}^{N_{a}} \int_{V_{j}^{a}} \rho_{j}^{a}\left(\dot{\mathbf{u}}^{\mathrm{T}} \dot{\mathbf{u}}\right) \mathrm{d} V_{j}^{a}+\frac{1}{2} \sum_{j=1}^{N_{\mathrm{se}}} \int_{V_{j}^{\mathrm{se}}} \rho_{j}^{\mathrm{se}}\left(\dot{\mathbf{u}}^{\mathrm{T}} \dot{\mathbf{u}}\right) \mathrm{d} V_{j}^{\mathrm{se}},
\end{aligned}
$$

where $\dot{u}_{1}, \dot{u}_{2}$, and $\dot{u}_{3}$ are the velocity components in the $\boldsymbol{x}, \boldsymbol{y}$, and $z$ directions; $\rho^{\text {st }}, \rho^{\text {a }}$, and $\rho^{\text {se }}$ are the mass densities of structure, piezoelectric actuators, and piezoelectric sensors, respectively. The virtual work of external forces is written as [16]

$$
\delta W^{\mathrm{ext}}=\sum_{j=1}^{N_{a}} v_{j}^{a} \delta q_{j}^{a}+\sum_{j=1}^{N_{\mathrm{se}}} v_{j}^{\mathrm{se}} \delta q_{j}^{\mathrm{se}}+\sum_{j=1}^{N_{f}} \delta u\left(x_{j}, y_{j}\right)^{\mathrm{T}} f_{j},
$$

where $v$ and $f$ are voltage that applied to piezoelectric patches and concentrated force; $\delta \mathbf{q}$ and $\delta \mathbf{u}$ are the variations of electric charge and mechanical displacement.

We can write Eq. (1) like the following expression as a function of generalized coordinates [16]:

$$
\begin{aligned}
\mathrm{PE}= & \frac{1}{2} \mathbf{U}^{\mathrm{T}} \mathbf{K}^{\mathrm{st}} \mathbf{U}+\frac{1}{2} \mathbf{U}^{\mathrm{T}} \mathbf{K}_{\mathbf{D}}^{\mathrm{se}} \mathbf{U}+\frac{1}{2} \mathbf{U}^{\mathrm{T}} \mathbf{K}_{\mathbf{D}}^{\mathrm{a}} \mathbf{U}-\mathbf{U}^{\mathrm{T}} \mathbf{K}_{\mathbf{U q}}^{\mathrm{se}} \mathbf{q}^{\mathrm{se}}-\mathbf{U}^{\mathbf{T}} \mathbf{K}_{\mathbf{U q}}^{\mathrm{a}} \mathbf{q}^{\mathrm{a}} \\
& +\frac{1}{2} \mathbf{q}^{\mathrm{se} \mathbf{T}} \mathbf{K}_{\mathbf{q q}}^{\mathrm{se}} \mathbf{q}^{\mathrm{se}}+\frac{1}{2} \mathbf{q}^{\mathrm{a} \mathbf{T}} \mathbf{K}_{\mathbf{q q}}^{\mathrm{a}} \mathbf{q}^{\mathrm{a}} .
\end{aligned}
$$

In this context, a means actuator and se means sensor. $\mathbf{U}$ is the generalized coordinate of the plate response, and $\mathbf{q}$ is the electrical generalized coordinate vector. $\mathbf{K}^{\text {st }}, \mathbf{K}_{\mathbf{D}}^{\text {se }}$, and $\mathbf{K}_{\mathbf{D}}^{\mathbf{a}}$ are, respectively, stiffness matrices of the structure, piezoelectric sensors, and piezoelectric actuators, $\mathbf{K}_{\mathbf{U q}}^{\mathbf{s e}}$ and $\mathbf{K}_{\mathbf{U q}}^{\mathbf{a}}$ are coupling matrices of sensors and actuators, and $\mathbf{K}_{\mathbf{q q}}^{\mathbf{s e}}$ and $\mathbf{K}_{\mathbf{q q}}^{\mathrm{a}}$ are capacitance matrices of sensors and actuators, respectively.

The kinetic energy can be rewritten as

$$
K E=\frac{1}{2} \dot{\mathbf{U}} \mathbf{M}^{\mathrm{st}} \dot{\mathbf{U}}+\frac{1}{2} \dot{\mathbf{U}} \mathbf{M}^{\mathrm{se}} \dot{\mathbf{U}}+\frac{1}{2} \dot{\mathbf{U}} \mathbf{M}^{\mathrm{a}} \dot{\mathbf{U}},
$$

where $\mathbf{M}^{\text {st }}, \mathbf{M}^{\text {se }}$, and $\mathbf{M}^{\mathbf{a}}$ are mass matrices of the plate, piezoelectric sensors, and piezoelectric actuators. 
The external work can be written as

$$
\delta W^{\text {ext }}=\delta \mathbf{q}^{\mathbf{a T}} \mathbf{B}_{\mathbf{V}}^{\mathbf{a}} \mathbf{V}^{\mathbf{a}}+\delta \mathbf{q}^{\text {seT }} \mathbf{B}_{\mathbf{V}}^{\text {se }} \mathbf{V}^{\text {se }}+\delta \mathbf{U}^{\mathbf{T}} \mathbf{F}_{\mathbf{c}},
$$

where $\mathbf{F}_{\mathbf{c}}$ is

$$
\mathbf{F}_{\mathbf{c}}=\sum_{j=1}^{N_{f}} \mathbf{N}_{\mathbf{u}}^{\mathbf{T}}\left(x_{j}, y_{j}\right) f_{j},
$$

and $\mathbf{B}_{\mathbf{V}}^{\mathbf{a}}$ and $\mathbf{B}_{\mathbf{V}}^{\text {se }}$ are identity matrices.

Utilizing Hamilton's principle, the governing equations for the plate vibration equipped with piezoelectric sensor and actuator patches can be derived:

$$
\begin{aligned}
& \mathbf{M U}+\mathbf{K}_{\mathbf{U U}} \mathbf{U}-\mathbf{K}_{\mathbf{U q}}^{\mathrm{se}} \mathbf{q}^{\mathrm{se}}-\mathbf{K}_{\mathbf{U q}}^{\mathbf{a}} \mathbf{q}^{\mathbf{a}}=\mathbf{F} \\
& -\mathbf{K}_{\mathbf{q} \mathbf{U}}^{\mathbf{s e}} \mathbf{U}+\mathbf{K}_{\mathbf{q q}}^{\mathbf{s e}} \mathbf{q}^{\mathbf{s e}}=\mathbf{B}_{\mathbf{v}}^{\mathrm{se}} \mathbf{v}(t)^{\mathbf{s e}} \\
& -\mathbf{K}_{\mathbf{q} \mathbf{U}}^{\mathbf{a}} \mathbf{U}+\mathbf{K}_{\mathbf{q q}}^{\mathbf{a}} \mathbf{q}^{\mathbf{a}}=\mathbf{B}_{\mathbf{v}}^{\mathbf{a}} \mathbf{v}(t)^{\mathbf{a}}
\end{aligned}
$$

where $\mathbf{K}_{\mathbf{U q}}=\mathbf{K}_{\mathbf{q U}}^{\mathbf{T}}, \mathbf{M}=\mathbf{M}^{\text {st }}+\mathbf{M}^{\mathbf{s e}}+\mathbf{M}^{\mathbf{a}}$, and $\mathbf{K}_{\mathbf{U U}}=\mathbf{K}^{\text {st }}+\mathbf{K}^{\mathbf{s e}}+\mathbf{K}^{\mathbf{a}} . \mathbf{M}$ and $\mathbf{K}_{\mathbf{U U}}$ are, respectively, the total mass and passive stiffness matrices of the system.

Equation (8) can be rewritten as below if we consider $\mathbf{v}(\mathbf{t})^{\mathbf{s e}}=0$ :

$$
\mathbf{M} \ddot{\mathbf{U}}+\mathbf{K U}=\Upsilon_{\mathbf{a}} \mathbf{v}(t)^{\mathbf{a}}+\mathbf{F}_{\mathbf{c}},
$$

where

$$
\Upsilon_{\mathbf{a}}=\mathbf{K}_{\mathbf{U q}}^{\mathrm{a}} \mathbf{K}_{\mathbf{q q}}^{\mathrm{a}-1} \mathbf{B}_{\mathbf{v}}^{\mathrm{a}}
$$

is the influence matrix of input voltage applied across actuator patches. The total active and passive matrix of system stiffness is expressed as follows:

$$
\mathbf{K}=\mathbf{K}_{\mathbf{U U}}-\mathbf{K}_{\mathbf{U q}}^{\mathrm{se}} \mathbf{K}_{\mathbf{q q}}^{\mathrm{se}-1} \mathbf{K}_{\mathbf{q U}}^{\mathrm{se}}-\mathbf{K}_{\mathbf{U q}}^{\mathrm{a}} \mathbf{K}_{\mathbf{q q}}^{\mathrm{a}-1} \mathbf{K}_{\mathbf{q U}}^{\mathrm{a}} .
$$

The current through the thickness of each sensor patch is equal to the time derivative of the electric charge accumulated on the surface of each sensor as given in Eq. (9):

$$
\mathbf{i}^{\mathbf{s e}}(t)=\frac{\mathrm{dq}^{\mathbf{s e}}(t)}{\mathrm{d} t} .
$$

When the used piezoelectric sensor is for sensing the strain rate, the current can be converted into the output open circuit sensor voltage [11]. Using Eqs. (9) and (14), the output sensor voltage can be expressed as

$$
\boldsymbol{\Phi}^{\mathrm{se}}(t)=\mathbf{G}_{\mathbf{c}} \mathbf{i}(t)=\mathbf{G}_{\mathbf{c}} \mathbf{K}_{\mathbf{q q}}^{\mathrm{se}-1} \mathbf{K}_{\mathbf{q} \mathbf{U}}^{\mathrm{se}} \dot{\mathbf{U}}=\mathbf{C} \dot{\mathbf{U}},
$$

where $\mathbf{G}_{\mathrm{c}}$ is the matrix of constant gains of the current amplifier and converts the sensor current vector to an output sensor voltage vector. Equation (15) is the sensor equation which transforms the strain rate to a voltage. The eigenvalue problem of Eq. (11) can be solved for determining the eigenvalues and eigenvectors. The orthonormality property must be satisfied as:

$$
\mathbf{H}^{\mathrm{T}} \mathbf{M H}=\mathbf{I}, \mathbf{H}^{\mathrm{T}} \mathbf{K H}=\Lambda_{\mathrm{n}}^{\mathbf{2}},
$$

where $\boldsymbol{n}$ is the number of the modes, $\boldsymbol{\Lambda}_{\mathbf{n}}^{2}=\operatorname{diag}\left(\left[\omega_{1}^{2} \omega_{2}^{2} \omega_{3}^{2} \ldots \omega_{n}^{2}\right]\right)$ is the eigenvalue matrix, and $\mathbf{H}$ is the eigenvector matrix. Modal coordinates are introduced as:

$$
\mathbf{U}=\mathbf{H R},
$$

where $\mathbf{R}$ is the generalized displacement vector.

By using modal coordinates and the addition of a structural damping ratio matrix, modal equations of motion and modal sensor equation can be obtained as [17]

$$
\ddot{\mathbf{R}}+2 \mathbf{Z} \Lambda_{\mathbf{n}} \dot{\mathbf{R}}+\Lambda_{\mathbf{n}}^{2} \mathbf{R}=\boldsymbol{\Upsilon}_{\mathbf{a}}^{\prime} \mathbf{v}(t)^{\mathbf{a}}+\mathbf{H}^{\mathbf{T}} \mathbf{F}_{\mathbf{c}}, \boldsymbol{\Phi}^{\mathrm{se}}(\mathbf{t})=\mathbf{C}^{\prime} \dot{\mathbf{R}}
$$


where $\boldsymbol{\Upsilon}_{\mathbf{a}}^{\prime}=\mathbf{H}^{\mathbf{T}} \boldsymbol{\Upsilon}_{\mathbf{a}}, \mathbf{C}^{\prime}=\mathbf{C H}$. $\mathbf{Z}$ is a diagonal matrix of modal damping ratios, $\boldsymbol{\Lambda}_{\mathbf{n}}$ is a diagonal matrix of natural frequencies, and $\Lambda_{\mathbf{n}}^{2}$ is a diagonal matrix which is equal to the square of natural frequencies. The matrices $\mathbf{Z}$ and $\boldsymbol{\Lambda}_{\mathbf{n}}$ are written as:

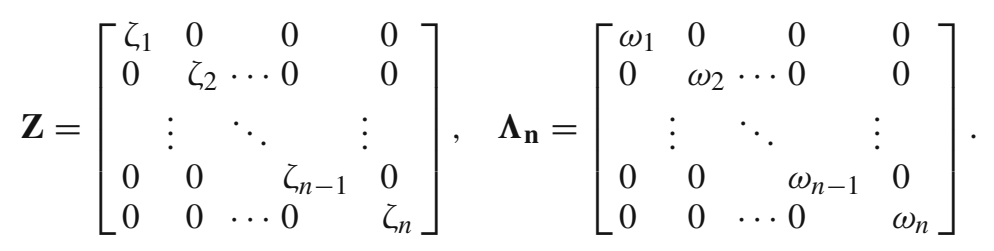

\section{Optimal locations of the piezoelectric sensors and actuators}

To determine the optimal piezoelectric sensor and actuator locations for a simply supported plate, a modified optimization criterion based on the concept of spatial $\mathbf{H}_{2}$ norm is used [18]. In this method, two criteria for determining the optimal placement of piezoelectric actuators and sensors are proposed, using spatial controllability/observability, and considering the effect of residual modes to reduce the spillover effect for the simply supported plate. Here, we consider a state of no in-plane extension and assume the plate is only in a condition of pure bending. With this assumption, the spatially discredited displacement field of the mid-plane is written as:

$$
u_{1}=0, u_{2}=0, u_{3}=\mathbf{N}_{\mathbf{u}_{3}} \mathbf{U}_{\mathbf{3}} .
$$

We assume that the voltages applied to the actuator patches are as follows:

$$
\mathbf{v}(t)^{\mathbf{a}}=\left[v(t)_{1}^{a} v(t)_{2}^{a} \ldots v(t)_{N_{a}}^{a}\right] .
$$

By the assumption that the initial conditions and external mechanical forces are zero, the transfer function of the plate resulting from Laplace transforming Eq. (18) can be written as:

$$
G(x, y, s)=\sum_{i=1}^{n} G_{i}(x, y, s)=\sum_{i=1}^{n} \frac{N_{u_{3}}^{i} \Upsilon_{a}^{i}}{s^{2}+2 \zeta_{i} \omega_{i} s+\omega_{i}^{2}} .
$$

Equation (22) is the transfer function from the voltages applied to the actuator patches, $\mathbf{v}(t)^{\mathbf{a}}$, to the plate pure bending, $u_{3} . \Upsilon_{a}^{i}$ is defined as:

$$
\Upsilon_{a}^{i}=\left[\left(K_{U q}^{a(1)}\right)_{i}\left(K_{q q}^{a(1)}\right)^{-1}\left(K_{U q}^{a(2)}\right)_{i}\left(K_{q q}^{a(2)}\right)^{-1} \cdots\left(K_{U q}^{a\left(N_{a}\right)}\right)_{i}\left(K_{q q}^{a\left(N_{a}\right)}\right)^{-1}\right],
$$

and $N_{u_{3}}^{i}$ is the $i$ th component of the vector $\mathbf{N}_{\mathbf{u}_{3}}$. Now, we are in a position to define the spatial $\mathcal{H}_{2}$ norm of a transfer function as [18-20]:

$$
\|G\|_{2}^{2}=\int_{-\infty}^{+\infty} \int_{0}^{l_{x s}} \int_{0}^{l_{y s}} \operatorname{trace}\{G(j \omega, x, y) * G(j \omega, x, y)\} \mathrm{d} y \mathrm{~d} x \mathrm{~d} \omega=\sum_{i=1}^{n}\left\|\tilde{G}_{i}\right\|_{2}^{2},
$$

where trace is defined to be the sum of the elements on the main diagonal of a matrix and

$$
\tilde{G}_{i}=\frac{\Upsilon_{a}^{i}}{s^{2}+2 \zeta_{i} \omega_{i} s+\omega_{i}^{2}}, \quad i=1, \ldots, n .
$$

We find the optimal location of the $j$ th piezoelectric actuator patch by defining the function $\psi_{i j}$ as:

$$
\psi_{i j}\left(x^{a(j)}, y^{a(j)}, \theta^{a(j)}\right)=\left\|\tilde{G}_{i}^{j}\right\|_{2}=\left|\left(K_{U q}^{a(j)}\right)_{i}\left(K_{q q}^{a(j)}\right)^{-1}\right|\left\|\frac{\Upsilon_{a}^{i}}{s^{2}+2 \zeta_{i} \omega_{i} s+\omega_{i}^{2}}\right\|, \quad i=1, \ldots, n,
$$

where $\left(x^{a(j)}, y^{a(j)}\right)$ and $\theta^{a(j)}$ are the corner coordinates of the $j$ th actuator patch and its orientation, respectively. The actuator orientation may change in the interval $(0 \leq \theta<\pi)$. In this study, the variables of the actuator locations that should be optimized are $\left\{\left(x^{a(j)}, y^{a(j)}, \bar{\theta}^{a(j)}\right)\right\}, j=1,2, \ldots, N_{a}$. The orientation of the patches is shown in Fig. 1. 
For enhancing the performance of feedback control, we should reduce the spillover effects. For achieving this purpose, it is necessary that with due consideration of the residual modes, we minimize the authority of the actuators over the high-frequency modes. Furthermore, we can enhance the performance of control with considering only the first $N_{C}$ modes and place the actuators at the locations that they provide a high authority over the first $N_{C}$ modes. The actuators optimal locations can be found by maximizing the following criterion:

$$
J_{a}=\frac{\sum_{j=1}^{N_{a}} \sqrt{\sum_{i=1}^{N_{C}} \psi_{i j}\left(x^{a(j)}, y^{a(j)}, \theta^{a(j)}\right)^{2}}}{\max \sum_{j=1}^{N_{a}} \sqrt{\sum_{i=1}^{N_{C}} \psi_{i j}\left(x^{a(j)}, y^{a(j)}, \theta^{a(j)}\right)^{2}}}-\lambda \frac{\sum_{j=1}^{N_{a}} \sqrt{\sum_{i=N_{C}}^{N_{C}+N_{R}} \psi_{i j}\left(x^{a(j)}, y^{a(j)}, \theta^{a(j)}\right)^{2}}}{\max \sum_{j=1}^{N_{a}} \sqrt{\sum_{i=N_{C}}^{N_{C}+N_{R}} \psi_{i j}\left(x^{a(j)}, y^{a(j)}, \theta^{a(j)}\right)^{2}}}
$$

where $\lambda$ is a weighting constant, and $N_{C}$ and $N_{R}$ are the numbers of controlled and residual modes.

Regarding that the spatial controllability is the same as the spatial $\mathrm{H}_{2}$ norm, for obtaining it, a limited number of modes are considered. For optimizing piezoelectric sensor locations, we rewrite Eq. (18) as:

$$
\boldsymbol{\Phi}^{\mathrm{se}}(t)=\mathbf{C}^{\prime} \dot{\mathbf{R}}=\mathbf{C} \dot{\mathbf{U}}=\sum_{i=1}^{n} C_{i} \dot{U}_{i}
$$

where $C_{i}$ is defined as:

$$
C_{i}=\left[G_{c}^{1}\left(K_{q q}^{\mathrm{se}(1)}\right)^{-1}\left(K_{U q}^{\mathrm{se}(1)}\right)_{i} G_{c}^{2}\left(K_{q q}^{\mathrm{se}(2)}\right)^{-1}\left(K_{U q}^{\mathrm{se}(2)}\right)_{i} \cdots G_{c}^{N_{\mathrm{se}}}\left(K_{q q}^{\mathrm{se}\left(N_{\mathrm{se}}\right)}\right)^{-1}\left(K_{U q}^{\mathrm{se}\left(N_{\mathrm{se}}\right)}\right)_{i}\right]^{\mathrm{T}} .
$$

We find the optimal location of the $j$ th piezoelectric sensor by defining the function $\Theta_{i j}$ as:

$$
\Theta_{i j}\left(x^{\mathrm{se}(j)}, y^{\mathrm{se}(j)}, \theta^{\mathrm{se}(j)}\right)=\left|C_{i}^{j}\right|=\left|G_{c}^{j}\left(K_{U q}^{\mathrm{se}(j)}\right)_{i}\left(K_{q q}^{\mathrm{se}(j)}\right)^{-1}\right|,
$$

where $\left(x^{s} e(j), y^{s} e(j)\right)$ and $\theta^{\operatorname{se}(j)}$ are the corner coordinates of the $j$ th sensor and its orientation, respectively. The orientation of sensors changes in the interval $(0 \leq \theta<\pi)$. In this work, the variables of sensor locations that should be optimized are as follows: $\left\{\left(x^{\operatorname{se}(j)}, y^{\operatorname{se}(\bar{j})}, \theta^{\operatorname{se}(j)}\right)\right\}, j=1,2, \ldots, N_{\mathrm{se}}$.

The optimal placement of sensors can be found by maximizing the following criterion:

$$
J_{\mathrm{se}}=\frac{\sum_{j=1}^{N_{\mathrm{se}}} \sqrt{\sum_{i=1}^{N_{C}} \Theta_{i j}\left(x^{\mathrm{se}(j)}, y^{\operatorname{se}(j)}, \theta^{\operatorname{se}(j)}\right)^{2}}}{\max \sum_{j=1}^{N_{\mathrm{se}}} \sqrt{\sum_{i=1}^{N_{C}} \Theta_{i j}\left(x^{\mathrm{se}(j)}, y^{\operatorname{se}(j)}, \theta^{\operatorname{se}(j)}\right)^{2}}}-\tilde{\lambda} \frac{\sum_{j=1}^{N_{\mathrm{se}}} \sqrt{\sum_{i=N_{C}}^{N_{C}+N_{R}} \Theta_{i j}\left(x^{\mathrm{se}(j)}, y^{\mathrm{se}(j)}, \theta^{\operatorname{se}(j)}\right)^{2}}}{\max \sum_{j=1}^{N_{\mathrm{se}}} \sqrt{\sum_{i=N_{C}}^{N_{C}+N_{R}} \Theta_{i j}\left(x^{\mathrm{se}(j)}, y^{\mathrm{se}(j)}, \theta^{\mathrm{se}(j)}\right)^{2}}} .
$$

It is observed that in this method the residual modes are considered and their influence on the optimization criterion is controlled by weighting constant $\tilde{\lambda}$. By comparing Eqs. (27) and (31), it is understood that for optimizing both sensor and actuator locations, the same procedure should be implemented.

\section{Application of GA for optimal location of piezoelectric devices}

In this study, the GA is utilized for optimizing the piezoelectric device locations on the flexible plate. A genetic algorithm is a means by which the machine can simulate the mechanism of natural selection. This operation is done by searching in the design space for finding the best solution. The genetic algorithm starts a search from a series of points, and for performing the search procedure, it does not require to the Jacobian of functions [21]. Many researchers for optimizing their work have used the genetic algorithm [22-25]. The terminology used for genetic algorithm is given in Table 1. Usually with the progress in the next generations, the algorithm converges to an asymptote point and the amount of fitness function improves. When the convergence is reached or the stopping criteria are satisfied, the search process stops. For optimization, a GA with the following configuration is considered in the work: population size: 200 , crossover rate: 0.8 , number of generations: 50 . Numerical simulations of the optimization process are analyzed in this section. We consider a simply supported rectangular plate for which the piezoelectric patches are attached to the both upper and lower surfaces. We suppose that the piezoelectric sensors and actuators are perfectly bonded to the surfaces. Tables 2, 3, and 4 give the geometrical and mechanical properties of materials that are used in this study. 
Table 1 Terminology for GA

\begin{tabular}{|c|c|}
\hline Terminology & Description \\
\hline Fitness function & The function that should be optimized [ $J$ in Eqs. (27) and (31)] \\
\hline Individual & $\begin{array}{l}\text { Any point that is utilized in the fitness function is an individual. The number of variables } \\
\text { for finding piezoelectric device positions and orientations is } 3 \times N_{\text {se }} \text { or } 3 \times N_{\mathrm{a}} \text { that are } \\
\text { equal to the number of individuals }\end{array}$ \\
\hline Population & $\begin{array}{l}\text { An array of individuals forms the population. If the size of population is } N_{i} \text { and the } \\
\text { number of variables or individuals are } 3 \times N_{\text {se }} \text { or } 3 \times N_{\mathrm{a}} \text {, then the population is shown } \\
\text { by } N_{i} \text { by } 3 \times N_{\text {se }} \text { or } N_{i} \text { by } 3 \times N_{\mathrm{a}} \text { matrices }\end{array}$ \\
\hline Beginning & Creating a casual population is the first step of GA \\
\hline Evaluation & The value of $J$ in Eqs. (27) and (31) to be evaluated for each of population \\
\hline Selection & Selects two parents having the largest value of the fitness function \\
\hline Crossover & Incorporates two parents that produce new children \\
\hline Mutation & $\begin{array}{l}\text { In order that this operator produces the children, it applies a random change in parents. } \\
\text { New children are placed in new population }\end{array}$ \\
\hline
\end{tabular}

Table 2 Geometrical parameters of the plate and the piezoelectric sensors and actuators

\begin{tabular}{lll}
\hline Parameter & Plate & Piezoelectric patch \\
\hline$x$-Length $(\mathrm{m})$ & 1 & 0.08 \\
$y$-Length $(\mathrm{m})$ & 0.5 & 0.04 \\
Thickness $(\mathrm{m})$ & 0.002 & 0.0001 \\
\hline
\end{tabular}

Table 3 Properties of sensors and actuators of PZT-5H [16]

\begin{tabular}{llll}
\hline Mechanical & & Electrical & Coupling \\
\hline$c_{11}^{D}=131.6(\mathrm{GPa})$ & $c_{22}^{D}=131.6(\mathrm{GPa})$ & $\beta_{33}^{\mathrm{s}}=1.48 \times 10^{8}(\mathrm{~m} / \mathrm{F})$ & $h_{13}=-2.72 \times 10^{9}(\mathrm{~N} / \mathrm{C})$ \\
$c_{12}^{D}=131.6(\mathrm{GPa})$ & $c_{66}^{D}=131.6(\mathrm{GPa})$ & & $h_{23}=-2.72 \times 10^{9}(\mathrm{~N} / \mathrm{C})$ \\
$\rho=7800\left(\mathrm{~kg} / \mathrm{m}^{3}\right)$ & & & \\
\hline
\end{tabular}

Table 4 Mechanical properties of the plate

\begin{tabular}{lll}
\hline Property & Symbol & Value \\
\hline Density & $\rho\left(\mathrm{kg} / \mathrm{m}^{3}\right)$ & 2770 \\
Young's modulus & $\mathrm{E}(\mathrm{GPa})$ & 70 \\
Poisson's ratio & $v$ & 0.3 \\
Damping ratio & $\zeta_{i}=0.0002 i=1, \ldots, n$ & \\
\hline
\end{tabular}

By using the Rayleigh-Ritz method and expanding the transverse displacement, $u_{3}(x, y, t)$ of the plate in terms of a time-dependent modal shape can be written as follows:

$$
u_{3}(x, y, t)=\sum_{\kappa=1}^{k} \sum_{l=1}^{l} N_{u_{3}}^{\kappa \iota}(x, y) U^{\kappa \iota}(t),
$$

where $k$ and $l$ are the total number of mode shapes in the longitudinal and lateral directions, respectively. $\kappa$ and $\iota$ are the numbers of half-waves in the longitudinal and lateral directions, and this series can be expressed as a matrix expansion:

$$
u_{3}=\left[N_{u_{3}}^{11} N_{u_{3}}^{21} N_{u_{3}}^{31} \ldots N_{u_{3}}^{k l}\right]\left[U^{11} U^{21} U^{31} \ldots U^{k l}\right]^{\mathrm{T}} .
$$

We define:

$$
\mathbf{N}_{\mathbf{u}_{3}}=\left[N_{u_{3}}^{11} N_{u_{3}}^{21} N_{u_{3}}^{31} \ldots N_{u_{3}}^{k l}\right] .
$$

For a simply supported plate, the modal shape functions that are used in this study are expanded in terms of double harmonic functions:

$$
N_{u_{3}}^{\kappa \iota}=\sin \sin \left(\frac{\kappa \pi x}{l_{x s}}\right) \sin \sin \left(\frac{\iota \pi y}{l_{y s}}\right),
$$


Table 5 First five natural frequencies

\begin{tabular}{llllll}
\hline Number of half wave $(\kappa, \iota)$ & $(1,1)$ & $(2,1)$ & $(3,1)$ & $(1,2)$ & $(2,2)$ \\
\hline Natural frequency $(\mathrm{Hz})$ & 23.88 & 38.22 & 62.11 & 81.23 & 95.57 \\
\hline
\end{tabular}
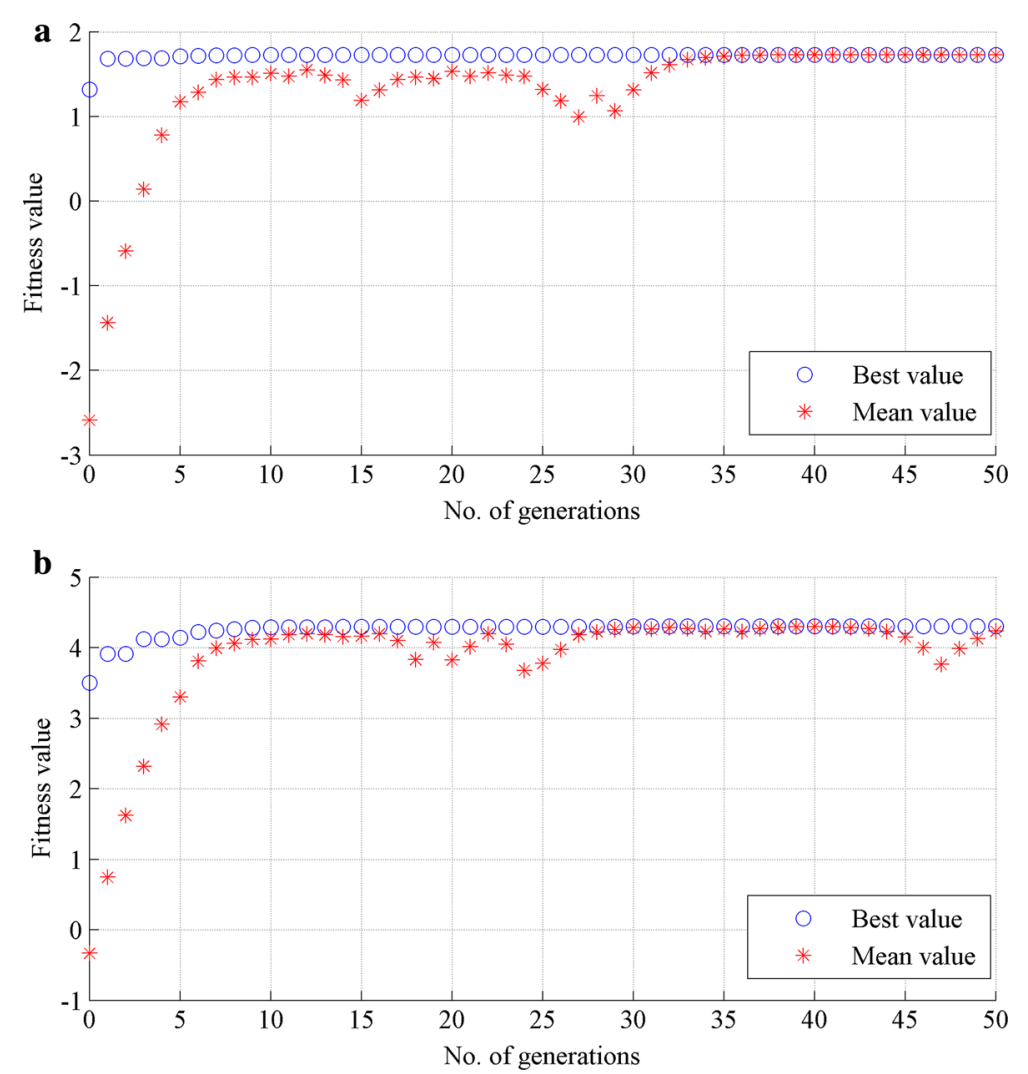

Fig. 2 Evolution of the mean and best values of fitness function for finding actuator/sensor locations when the first two modes are controlled: a one actuator/sensor, $\mathbf{b}$ a pair of actuator/sensor

where $l_{x s}$ and $l_{y s}$ are the length and width of the rectangular plate. For the purpose of vibration control, only the first five modes are considered, and their natural frequencies are presented in Table 5.

There are several examples considered here for showing the influence of the GA that is used for locating the piezoelectric devices attached to a thin plate. In the first two simulations, the first two lowest modes are considered that have the highest controllability and observability, and else, the remaining three modes are considered as the residual modes. Because the fitness functions for finding sensor and actuator locations are similar, herein we only discuss how to find the sensor locations. In Fig. 2a, the best and mean values of the fitness function for finding the optimal location of one sensor attached to the plate are shown. As can be seen, the best value for the objective function is equal to 1.68. The optimal corner point coordinates of one sensor location are $(0.657 \mathrm{~m}, 0.206 \mathrm{~m})$, and its orientation in this point is 52 degrees. The best and mean fitness function values for finding the optimal locations of two sensors are indicated in Fig. $2 \mathrm{~b}$.

In the first generations, the best value of fitness function is improved rapidly, that is because of being too far away from the optimal point. In the other simulations of this section, the first three lower modes are controlled and the remaining two modes are considered as residual modes. The evolutions of the best and mean values of the fineness function are presented in Fig. 3. The optimal positions of these simulations are listed in Table 6. For active vibration control, a negative velocity feedback control algorithm is used. This feedback strategy increases the depreciation rate; thus, it is an effective way for reducing the oscillatory amplitude. A simple algorithm of this type is used in such a way that the actuator voltage can be obtained with an amplifier gain and a change in polarity on the sensor voltage as follows:

$$
\mathbf{v}(t)^{\mathbf{a}}=-\mathbf{G}_{\mathbf{C o}} \Phi^{\mathbf{s e}}(t)
$$



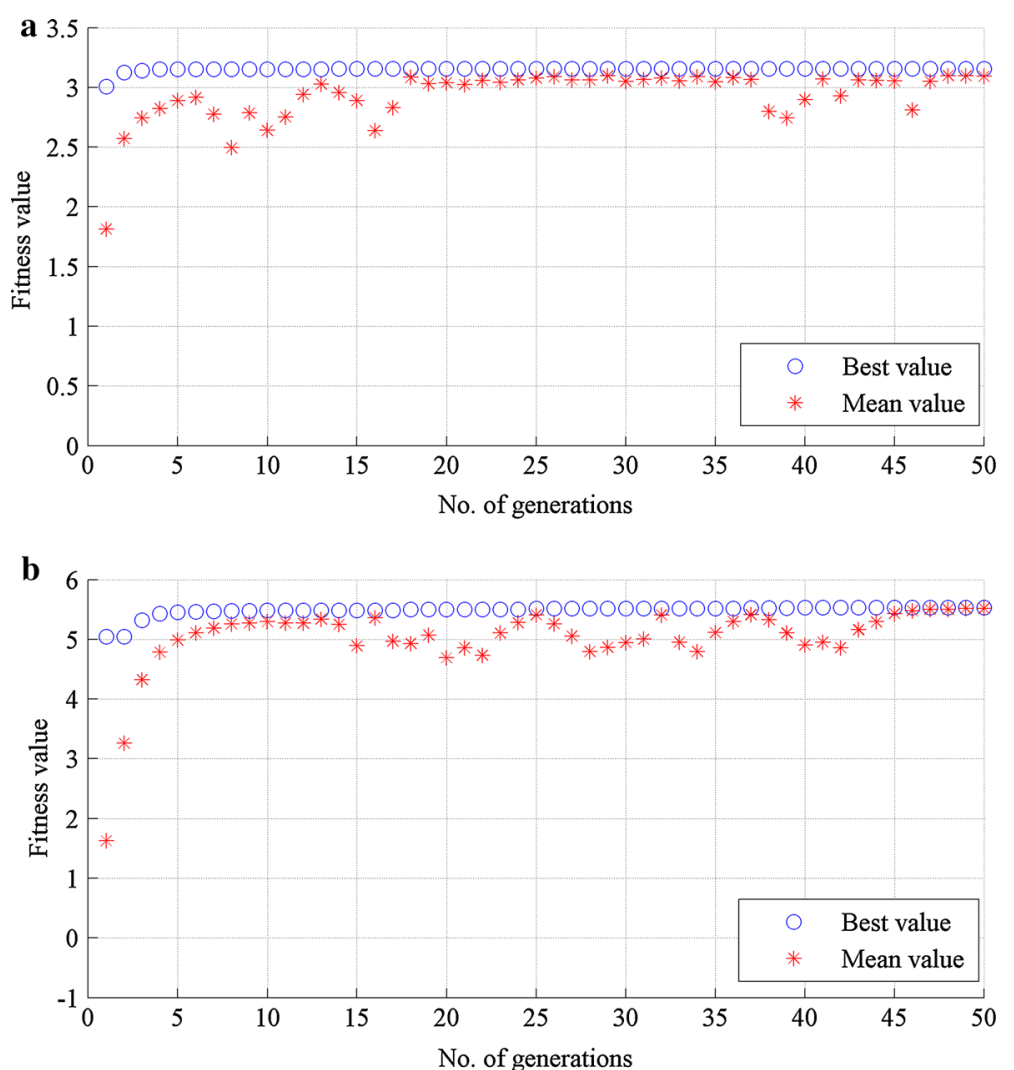

Fig. 3 Evolution of the mean and best values of fitness function for finding actuator/sensor locations when the first three modes are controlled: $\mathbf{a}$ one actuator/sensor $\mathbf{b}$ a pair of actuator/sensor

Table 6 Optimized location of piezoelectric patches attached to the rectangular plate

\begin{tabular}{|c|c|c|c|}
\hline Controlled modes & & Modes 1 and 2 & Modes 1,2 , and 3 \\
\hline Number of patches & $\begin{array}{l}1 \\
2\end{array}$ & $\begin{array}{l}0.6577 \mathrm{~m}, 0.2062,52.00^{\circ} \\
0.6739 \mathrm{~m}, 0.2059,72.68^{\circ} \\
0.3781 \mathrm{~m}, 0.2513,155.53^{\circ}\end{array}$ & $\begin{array}{l}0.4475 \mathrm{~m}, 0.2155 \mathrm{~m}, 103.0^{\circ} \\
0.4819 \mathrm{~m}, 0.2275 \mathrm{~m}, 123.33^{\circ} \\
0.4753 \mathrm{~m}, 0.1527 \mathrm{~m}, 86,61^{\circ}\end{array}$ \\
\hline
\end{tabular}

where $\mathbf{G}_{\mathbf{C o}}$ is a matrix of gains of the amplifier that is used in the feedback control.

\section{Results and discussion}

In this section, several simulations are presented for illustrating the influence of optimization procedure and active control algorithm. In the first simulation, the first two lower modes should be controlled and the remaining three modes are considered as residual modes. For exciting a larger number of system mode shapes, we have applied the step function concentrated load at a location off-center of the plate. The frequency responses of the plate, when excited by a concentrated force, and located at $x=0.43 \mathrm{~m}$ and $y=0.21 \mathrm{~m}$ for different number of sensors and actuators, are plotted in Fig. 4. As can be seen, the optimization procedure is more effective for the amplitude vibration reduction as contributed to arbitrary location of actuators/sensors. By locating the actuators and sensors in the optimal locations, more damping is developed in the plate. When the sensors are located at random locations, we could not observe the first two modes in the response, and when the actuators are located at random locations, the maximum mechanical energy cannot be transmitted to the first two modes. However, if the sensors are located at the optimal locations, the contributed modes could be observed. Furthermore, if the actuators are located at the optimal locations, the maximum mechanical energy could transmit to contribute modes and the amplitude of vibration would reduce more. 

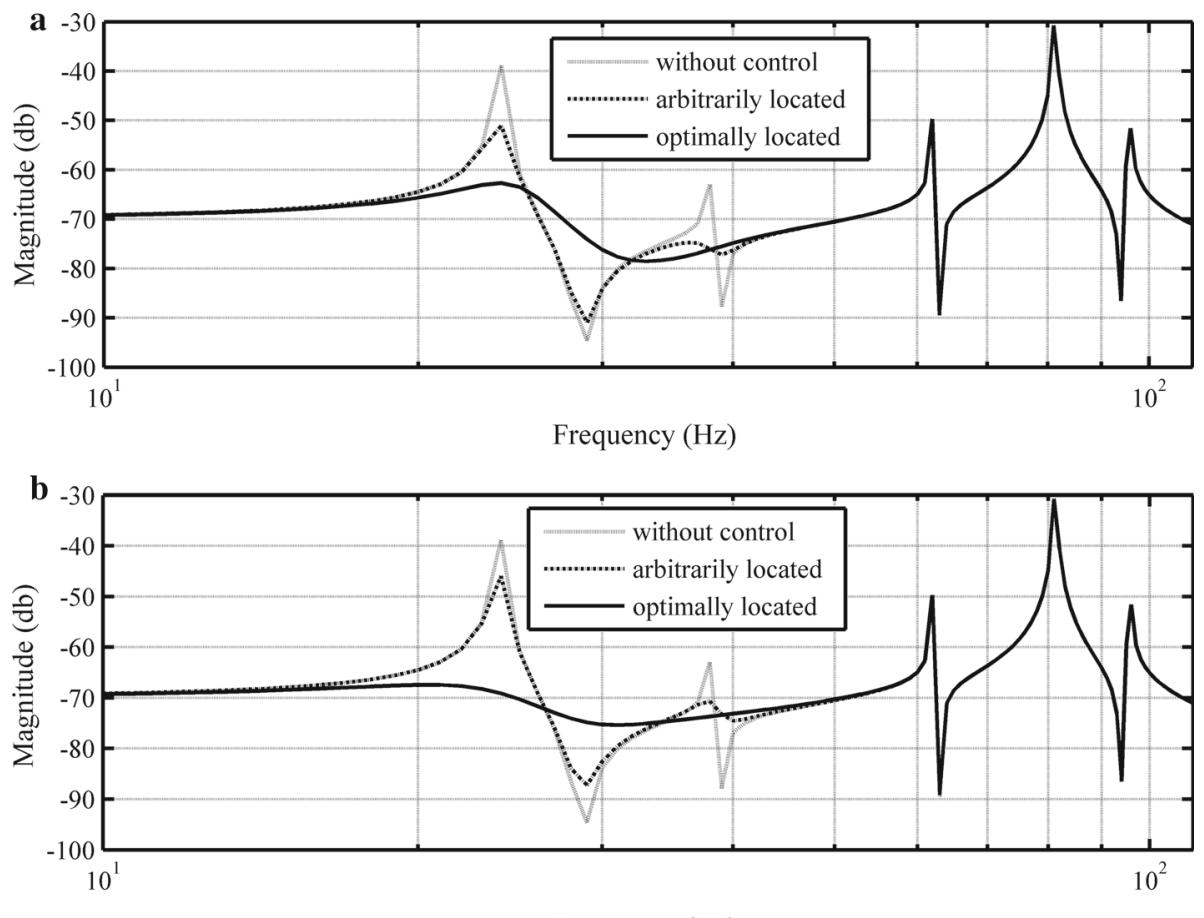

Frequency $(\mathrm{Hz})$

Fig. 4 Frequency response of the plate controlled by the piezoelectric actuator/sensor patches when the first two modes are controlled: a one actuator/sensor $\mathbf{b}$ a pair of actuator/sensor
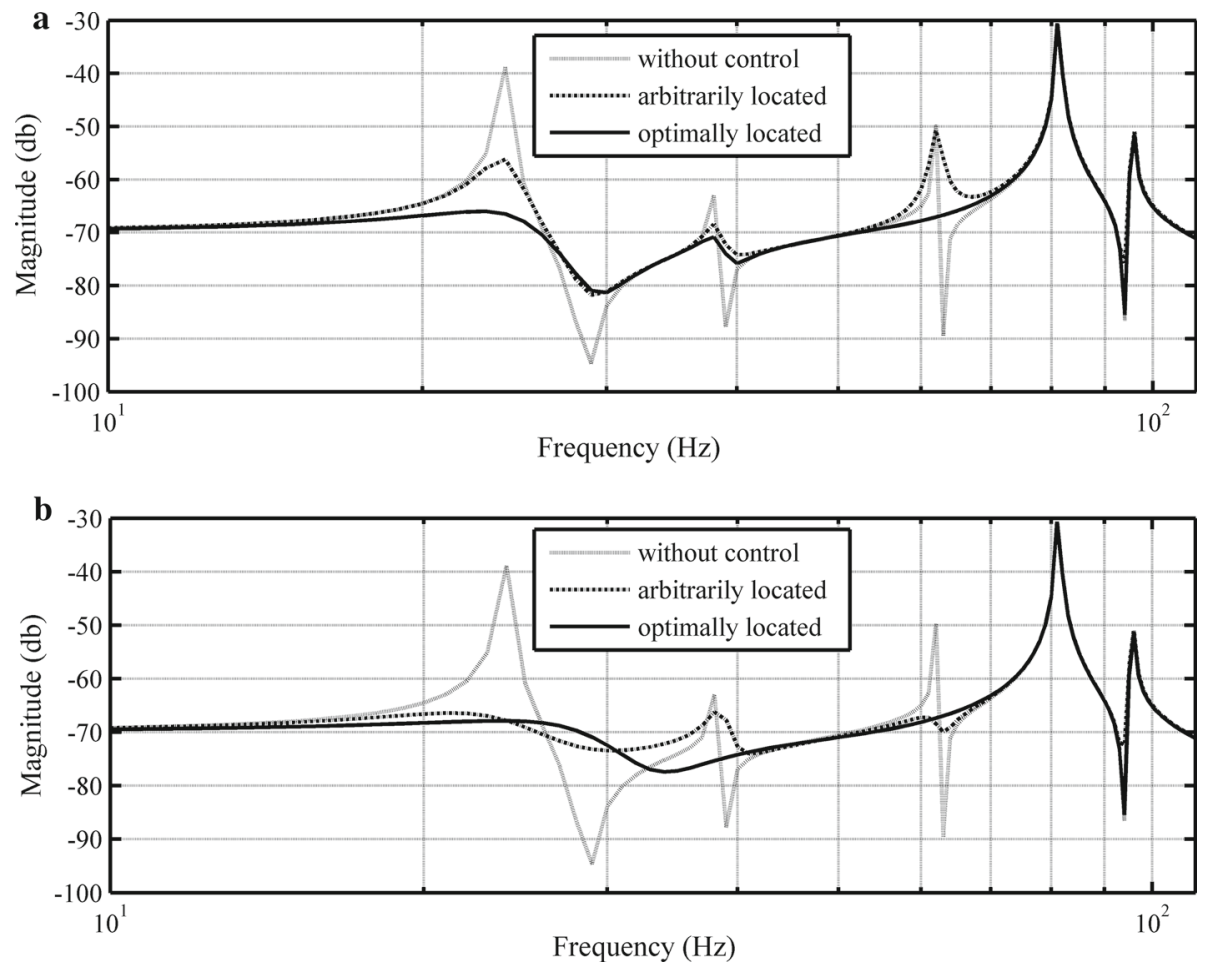

Fig. 5 Frequency response of the plate controlled by the piezoelectric actuator/sensor when the first three modes are controlled: a one actuator/sensor b two actuator/sensor 


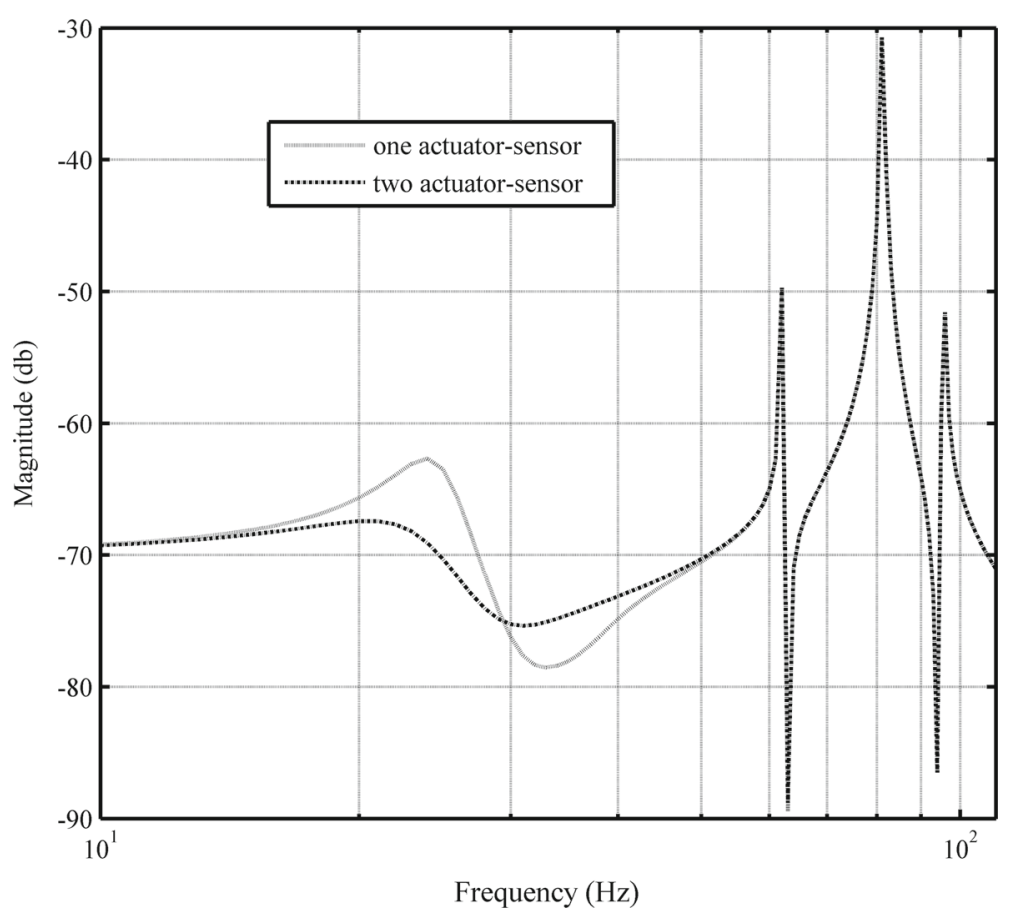

Fig. 6 Comparison of frequency response of the plate controlled by different number of piezoelectric actuator/sensor

In the second simulation, the first three lower modes must be controlled and the remaining two modes are residual modes. The frequency responses of the plate, excited by a concentrated force, and located at $x=0.43 \mathrm{~m}$ and $y=0.21 \mathrm{~m}$, for one and two number of sensors and actuators, are presented in Fig. 5. As can be seen, the optimization criteria are effective in having more damping effect. In Fig. 6, the influence of the number of sensors and actuators is compared in the control of vibration. In this simulation, the sensors and actuators are located in the optimal positions and first two lower modes must be controlled. It can be concluded that when a pair of actuators and sensors are used, the damping effect increases and the amplitude vibration of the plate reduces more.

\section{Conclusions}

In the present study, the active vibration control and optimal position of piezoelectric patches attached to a thin plate were analyzed. For deriving the equation of motion and sensor output equation, the Hamilton's principle and the Rayleigh-Ritz method were used. In the next step, the optimal positions and orientations of the piezoelectric actuators and sensors attached to a rectangular plate were determined based on the concept of spatial controllability/observability and considering residual modes, for reducing the spillover effect. A GA was utilized for optimizing the locations and orientations of the piezoelectric devices. The results indicated that by locating the piezoelectric sensors and actuators in the optimal positions, the damping effect could be increased and the amplitudes of plate vibration were reduced more effectively. Furthermore, in general the active vibration control was effective in the vibration control of the thin plate.

Acknowledgments The research leading to these results has received funding from the People Programme (Marie Curie Actions) of the European Union's Seventh Framework Programme FP7/2007-2013/ under REA Grant Agreement No. PITN-GA-2013606878 .

Open Access This article is distributed under the terms of the Creative Commons Attribution 4.0 International License (http:// creativecommons.org/licenses/by/4.0/), which permits unrestricted use, distribution, and reproduction in any medium, provided you give appropriate credit to the original author(s) and the source, provide a link to the Creative Commons license, and indicate if changes were made. 


\section{References}

1. Zhang, Y.H., Xie, S.L., Zhang, X.N.: Vibration control of a simply supported cylindrical shell using a laminated piezoelectric actuator. Acta Mech. 196, 87-101 (2008)

2. Sarangi, S.K., Ray, M.C.: Active damping of geometrically nonlinear vibrations of laminated composite plates using vertically reinforced 1-3 piezoelectric composites. Acta Mech. 222, 363-380 (2011)

3. Kumar, K.R., Narayanan, S.: Active vibration control of beams with optimal placement of piezoelectric sensors/actuator pairs. Smart Mat. Struct. 17, 055008 (2008)

4. Bruant, I., Coffignal, G., Le'ne', F., Verge', M.: A methodology for determination of piezoelectric actuator and sensor location on beam structures. J. Sound Vib. 243, 862-882 (2001)

5. Hac, A., Liu, L.: Sensor and actuator location in motion control of flexible structures. J. Sound Vib. 167, $239-261$ (1993)

6. Qiu, Z.-C., Zhang, X.-M., Wu, H.-X., Zhang, H.-H.: Optimal placement and active vibration control for piezoelectric smart flexible cantilever plate. J. Sound Vib. 301, 521-543 (2007)

7. Sadri, A.M., Wright, J.R., Wynne, R.J.: Modelling and optimal placement of piezoelectric actuators in isotropic plates using genetic algorithms. Smart Mat. Struct. 8, 490-498 (1999)

8. Bruant, I., Gallimard, L., Nikoukar, S.: Optimal piezoelectric actuator and sensor location for active vibration control, using genetic algorithm. J. Sound Vib. 329, 1615-1635 (2010)

9. Han, J.H., Lee, I.: Optimal placement of piezoelectric sensors and actuators for vibration control of a composite plate using genetic algorithms. Smart Mat. Struct. 8, 257-267 (1999)

10. Halim, D., Reza Moheimani, S.O.: An optimization approach to optimal placement of collocated piezoelectric actuators and sensors on a thin plate. Mechatronics 13, 27-47 (2003)

11. Yang, S., Lee, Y.: Optimization of noncollocated sensor/actuator location and feedback gain in control systems. Smart Mat. Struct. 2, 96-102 (1993)

12. Kumar, R., Mishra, B.K., Jain, S.C.: Static and dynamic analysis of smart cylindrical shell. Finite Elem. Anal. Des. 45, 1324 (2008)

13. Kwak, M.K., Heo, S.: Active vibration control of smart grid structure by multiinput and multioutput positive position feedback controller. J. Sound Vib. 304, 230-245 (2007)

14. Yiqi, M., Yiming, F.: Nonlinear dynamic response and active vibration control for piezoelectric functionally graded plate. J. Sound Vib. 329, 2015-2028 (2010)

15. He, X.Q., Ng, T.Y., Sivashanker, S., Liew, K.M.: Active control of FGM plates with integrated piezoelectric sensors and actuators. Int. J. Solids Struct. 38, 1641-1655 (2001)

16. Leo, D.J.: Engineering Analysis of Smart Material Systems. Wiley, New York (2007)

17. Inman, D.J.: Vibration with Control. Wiley, New York (2006)

18. Reza Moheimani, S.O., Halim, D., Fleming, A.J.: Spatial Control of Vibration Theory and Experiments. World Scientific, New York (2002)

19. Reza Moheimani, S.O., Fu, M.: Spatial $\mathrm{H}_{2}$ norm of flexible structures and its application in model order selection. In: Proceedings of the 37th IEEE Conference on Decision \& Control, pp. 3623-3624, Tampa, USA (1998)

20. Reza Moheimani, S.O., Ryall, T.: Considerations in placement of piezoceramic actuators that are used in structural vibration control. In: Proceedings of the 38th IEEE Conference on Decision \& Control, pp. 1118-1123, Phoenix, USA (1999)

21. Sivanandam, S.N., Deepa, S.N.: Introduction to Genetic Algorithms. Springer, New York (2008)

22. Yang, Y., Jin, Z., Kiong Soh, C.: Integrated optimal design of vibration control system for smart beams using genetic algorithms. J. Sound Vib. 282, 1293-1307 (2005)

23. Liu, W., Gao, W.C., Sun, Y., Xu, M.J.: Optimal sensor placement for spatial lattice structure based on genetic algorithms. J. Sound Vib. 317, 175-189 (2008)

24. Hasancebi, H., Erbatur, F.: Layout optimization of trusses using improved GA methodologies. Acta Mech. 146, 87-107 (2001)

25. Chiba, R., Sugano, Y.: Optimisation of material composition of functionally graded materials based on multiscale thermoelastic analysis. Acta Mech. 223, 891-909 (2012) 